\section{THU0289 B-CELL ACTIVATING FACTOR GENE EXPRESSION IN URINARY SAMPLE AND RENAL BIOPSY FOR MONITORING DISEASE ACTIVITY IN LUPUS NEPHRITIS}

S. Retamozo ${ }^{1,2}$, L. Mas ${ }^{3}$, M.J. Haye Salinas ${ }^{4}$, V. Saurit ${ }^{4}$, F. Caeiro ${ }^{4}$, A. Diller ${ }^{5}$ J. De La Fuente ${ }^{6}$, M. Angelina ${ }^{7}$, N.R. Benzaquen ${ }^{4}$, J.P. Pirola ${ }^{4}$, A. Alvarellos ${ }^{4}$ T. Alvarellos ${ }^{3} .{ }^{1}$ Rheumatology, Hospital Privado Universitario de Córdoba; ${ }^{2}$ INICSA, CONICET; ${ }^{3}$ Molecular Biology; ${ }^{4}$ Rheumatology; ${ }^{5}$ Pathology;

${ }^{6}$ Nephrology, Hospital Privado Universitario de Córdoba; ${ }^{7}$ Nephrology, Hospital Raul A Ferreyra, Cordoba, Argentina

Objectives: To evaluate BLyS as biomarker in disease activity in urinary sample and renal biopsy from patients with $\mathrm{LN}$.

Methods: Retrospective study. Between June 2009 and October 2013, 32 patients with SLE and LN fullfilling SLE classification criteria of ACR 1997 were included. The renal biopsies were evaluated according to the ISN/RPS classification system. The gene expression levels of BLyS were quantified using Quantitative Real Time PCR (QPCR). The relative quantification method was used for analysis, where Ct was normalized to an endogenous control $\beta 2$ Microglobulina ( $\beta 2 \mathrm{M})(\triangle \mathrm{Ct} \mathrm{BLyS}$ ). The data expressed as $\Delta \mathrm{Ct}$ are inversely proportional to gene expression level. The value of BLyS is expressed as median (M) and interquartile range (IQR) for filing a non-normal distribution.

Results: $26(81.3 \%)$ patients were female with a mean age at diagnosis of $26.9 \pm 13$ years and $31.9 \pm 29$ years at the time of renal biopsy. The SLEDAI at the time of biopsy was 10.5 (IQR $0-15.7$ ) and SLICC $\geq 1$ in $13(32.5 \%$ ), hypocomplementemia $13 / 31(41.9 \%)$ and positive DNA in 11/29 (37.9\%) patients. Biopsies from patients with proteinuria $\geq 0.5$ and renal failure (RF) $(n=23,71.9 \%)$, proteinuria isolated $(n=14,43.8 \%), L N$ remission. The value of the BLyS gene expression in renal biopsy was 8.09 (IQR 7.37-9.16) and BLyS in urinary sample was 6.45 (IQR 5.62-7.76).

Conclusions: BLyS detection in urinary samples could be a potential biomarker for predicting lupus nephritis activity. Our data confirm that the BLyS as urinary biomarker is present in patients with active renal disease especially in patients with proliferative glomerulonephritis.

Disclosure of Interest: None declared

DOI: 10.1136/annrheumdis-2017-eular.4523

\section{THU0290 CLINICAL BACKGROUND FACTORS RELATED TO SILENT OSTEONECROSIS OF THE FEMORAL HEAD UPON INITIATION OF STEROID THERAPY IN PATIENTS WITH SYSTEMIC LUPUS ERYTHEMATOSUS}

T. Kuroda ${ }^{1}$, N. Tanabe ${ }^{2}$, H. Sato ${ }^{1}$, T. Nakatsue ${ }^{1}$, Y. Wada ${ }^{1}$, M. Nakano ${ }^{3}$ I. Narita ${ }^{1} .{ }^{1}$ Division of Clinical Nephrology and Rheumatology, Niigata University Graduate School of Medical and Dental Sciences; ${ }^{2}$ Department of Health and Nutrition, Faculty of Human Life Studies, University of Niigata Prefecture: ${ }^{3}$ Department of Medical Technology, School of Health Sciences, Faculty of Medicine, Niigata University, Niigata, Japan

Background: Osteonecrosis of the femoral head (ONF) occurs frequently (3$40 \%$ in patients who receive corticosteroid therapy for SLE. MRI can accurately visualize pathological abnormalities of the femoral head, being much more sensitive than plain radiography. To analyze the risk factors associated with steroid-induced ONF, the best approach would be to examine early changes in the femoral head using MRI and any early clinical events attributable to steroid therapy. For treatment of SLE, a number of strategies may be selected according to the clinical conditions of patients, and treatments may differ slightly among hospitals. Therefore, for better clarification of the background factors associated with ONF, the optimum approach would be to analyze the treatment strategy, selection of steroid, initial dose of steroid and drugs used together with steroid in a cohort of patients treated at a single hospital.

Objectives: To clarify the factors related to silent ONF in patients with SLE treated at a single institution.

Methods: One hundred six patients (12 males and 94 females) with SLE were selected on the basis of having been newly diagnosed and requiring high-dose prednisolone, including pulse therapy with methylprednisolone, as the initial treatment. All the patients initially underwent plain radiography and MRI at the start of corticosteroid treatment to detect any early changes in the femoral head, and subsequent examinations were performed three months later. Laboratory parameters were evaluated at the start of steroid treatment and one month thereafter. All statistical analyses were performed with SPSS v. 13 (SPSS Inc., Chicago, IL, USA). Differences demonstrated by 2 -tailed tests were considered statistically significant at $P$ (two-sided) $<0.05$, and marginally significant at $P=0.05-0.10$.

Results: By three months after the start of corticosteroid treatment, asymptomatic ONF was diagnosed by MRI in 30 patients $(28.3 \%)$, being bilateral in 17 and unilateral in 13. Serological activity (C3, C4, CH50 and anti-ds DNA antibody), renal function (eGFR, serum creatinine and urinary protein), anti-phospholipid antibodies, and SLEDAI were not correlated with asymptomatic ONF. BMI, BSA, and the initial dose of prednisolone per unit body weight, BMI and BSA were also not correlated with asymptpmatic ONF. No preventive effect of ONF was observed by pretreatment with statins. However, patients with angiitis and a elevated total cholesterol level at 4 weeks after the start of steroid treatment tended to show a higher incidence of ONF. Patients with a higher triglyceride level both before and 4 weeks after the start of steroid treatment showed a significantly higher frequency of asymptomatic ONF $(\mathrm{P}<0.001)$.

Conclusions: Asymptomatic ONF is common in patients with SLE. A high triglyceride level is a significant risk factor for ONF, and large epidemiologic surveys would help to shed light on early events such as silent ONF in patients receiving steroid therapy.

Acknowledgements: This study was supported by a research grant from the Research Committee on Idiopathic Osteonecrosis of the Femoral Head of the Ministry of Health, Labour, and Welfare of Japan.

Disclosure of Interest: None declared

DOI: 10.1136/annrheumdis-2017-eular.2249

\section{THU0291 GENDER INFLUENCE ON CLINICAL, BIOLOGICAL AND IMMUNOLOGICAL ASPECTS OF SYSTEMIC LUPUS ERYTHEMATOSUS}

T. Ben Salem, M. Tougorti, I. Naceur, I. Ben Ghorbel, M. Lamloum M.H. Houman. Internal Medicine, Rabta university hospital, Tunis, Tunisia

Background: Systemic lupus erythematosus (SLE) is more frequent in women but seems to be more severe in men.

Objectives: The aim was to study gender influence on clinical, biological and immunological features of SLE

Methods: It's a retrospective study conducted in an internal medicine department. Patients with systemic lupus erythematosus (ACR revised criteria) were included. Data were recorded and compared using SPSS. Variables with a $p \leq 0.05$ were considered to be statistically significant.

Results: A total of 246 SLE patients were included; 224 female and 19 male (sex ratio $F / M$ was 11.78). Mean ages at disease onset and at SLE diagnosis were comparable for men and women respectively $35.63 \pm 14.31$ vs $32.7 \pm 13.36$ years and $35.8 \pm 14.3$ vs $34.5 \pm 13.6$ years.

SLE diagnosis was made earlier in men with an average delay (from first sign of the disease to diagnosis) of 6.1 months vs 21.4 months ( $p=0.02)$. Clinically, photosensitivity was significantly more frequent in women ( $81.4 \%$ vs $58.8 \%$; $p=0.03$ ). Women complained from alopecia more frequently than men but the difference was not statistically significant $(35.1 \%$ vs $14.3 \% ; p=0.09)$. Arthritis were two times more frequent in women $(50.7 \%$ vs $25 \% ; p=0.04)$. Lupus nephritis us well us lupus pancreatitis were significantly more frequent in men, respectively $66.7 \%$ vs $41.6 \%(p=0.039)$ and $11.1 \%$ vs $1.4 \%(p=0.047)$. There were no differences according to gender in neurological involvement and seritis. No significant differences were observed between men and women concerning hematological disorders. Anti-DNA, anti-Sm, anti-RNP, anti-SSA, anti-SSB, anti-cardiolipin and anti-B2GP1 antibodies frequencies were similar in both genders.

Conclusions: SLE diagnosis was made earlier in men than women, this could be explained by more severe disease in men [1]. However this hypothesis has been highly controversial [2]. Cutaneous and joints manifestations seem to be more frequent in women whereas serious manifestations like lupus nephritis and neurological involvements were more frequent in men [3-4]. In our study, only lupus nephritis and pancreatitis were more frequent in men.

\section{References:}

[1] Andrade RM, Alarcon GS, Fernandez M, Apte M, Vila LM, Reveille JD. Accelerated damage accrual among men with systemic lupus erythematosus: XLIV. Results from a multiethnic US cohort. Arthritis Rheum. 2007:56:622-30.

[2] Murphy G, Isenberg D. Effect of gender on clinical presentation in systemic lupus erythematosus. Rheumatology (Oxford). 2013:52:2108-15.

Abstract THU0289 - Table 1. BLyS gene expression in urinary sample and renal biopsy according to clinical and histological findings

\begin{tabular}{|c|c|c|c|c|}
\hline Variables & $\Delta$ Ct BLyS Urinary & $\mathrm{p}$ & $\Delta$ Ct BLyS Biopsy & $\mathrm{p}$ \\
\hline SLEDAI $=0 /$ SLEDAI $\geq 6$ & $7.52(6.59-11.19) / 5.94(5.52-7.08)$ & 0.04 & $8.03(6.90-10.20) / 8.15(7.35-9.10)$ & 0.82 \\
\hline $\mathrm{Bx} \mathrm{LN}$ in remission/LN active & $7.52(6.59-11.19) / 5.94(5.52-7.08)$ & 0.04 & $8.03(6.90-10.20) / 8.15(7.35-9.10)$ & 0.82 \\
\hline MDRD $\geq 60 /$ MDRD $\leq 60$ & $6.76(6.018 .12) / 5.60(5.16-7.19)$ & 0.04 & $8.03(6.95-8.95) / 8.41(7.45-13.40)$ & 0.42 \\
\hline Proteinuria $\leq 0.5 /$ Proteinuria $\geq 0.5$ & $7.52(6.59-11.19) / 5.94(5.52-7.08)$ & 0.04 & $8.03(6.90-10.20) / 8.15(7.35-9.10)$ & 0.82 \\
\hline Without tubular atrophy in $\mathrm{Bx} /$ Tubular atrophy in $\mathrm{Bx}$ & $7.83(6.43-11.67) / 6.14(5.51-7.24)$ & 0.03 & $8.16(6.44-8.52) / 8.09(7.42-10.55)$ & 0.53 \\
\hline
\end{tabular}

Abstract THU0289 - Table 2. BLyS gene expression in urinary sample and renal biopsy according to the classification of LN

\begin{tabular}{|c|c|c|c|c|c|}
\hline & Class I/Normal Bx & Class II Bx & Class IV Bx & Class V/VI Bx & $\mathrm{P}$ \\
\hline$\Delta$ Ct BLyS Renal Biopsy & $7.56(6.50-7.56)$ & $8.41(7.36-10.37)$ & $7.95(7.37-10.04)$ & $8.28(7.37-11.80)$ & 0.88 \\
\hline$\Delta$ Ct BLyS Urinary ${ }^{\star \star \star}$ & $7.28(9.95-7.28)$ & $8.41(7.36-10.37)$ & $5.65(5.45-6.40)$ & $6.34(5.74-7.32)$ & 0.003 \\
\hline
\end{tabular}

${ }^{* * *} \mathrm{p}<0.05$ Class I/normal with class IV, Class II with IV, class II with V/VI. 
[3] Tan TC, Fang H, Magder LS, Petri MA. Differences between male and female systemic lupus erythematosus in a multiethnic population. J Rheumatol. 2012;39:759-69.

[4] Boodhoo KD, Liu S, Zuo X. Impact of sex disparities on the clinical manifestations in patients with systemic lupus erythematosus: A systematic review and meta-analysis. Medicine. 2016;95(29):e4272.

Disclosure of Interest: None declared

DOI: 10.1136/annrheumdis-2017-eular.2323

\section{THU0292 DIAGNOSTIC ACCURACY OF ULTRASOUND AND ULTRASONOGRAPHIC FEATURES OF SALIVARY GLANDS IN PATIENTS WITH PRIMARY SJOGREN'S SYNDROME}

V. Milic, G. Radunovic, N. Damjanov. Institute of Rheumatology, Belgrade, Serbia

Objectives: To analysis ultrasonography (US) changes of salivary glands (SG) in patients with primary Sjogren's syndrome (pSS) and assessment of their accuracy for diagnosis pSS.

Methods: This study included 205 pSS patients (mean age 53.9 \pm 11.5 , disease

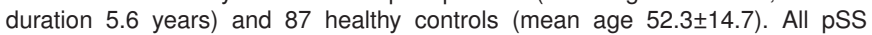
patients fulfilled the AECG diagnostic criteria. The disease activity was measured by EULAR SS disease activity index (ESSDAI). Parotid and submandibular glands on both sides were assessed for size, parenchymal echogenicity and inhomogeneity, posterior glandular border and presence of intraglandular lymph nodes. Inhomogeneity of the salivary glands were graded according to the De Vita scoring system [0) homogenous glands; 1 ) mild inhomogeneous - isolated hypoechogenic areas; 2) evident inhomogeneous - scattered hypoechogenic areas, and/or multiple punctate or linear densities; 3) grossly inhomogeneous - large or confluents hypoechogenic areas, and/or to linear densities, and/or multiple cysts. The global SGUS score (0-6) was the sum of the scores of each pair of salivary glands. Statistical analysis was performed by SPSS v16. Data were compared using $t$-test, $\chi^{2}$ test and Mann-Whitney $U$ test. The optimal cut-off value for SGUS score was calculated as the area under the receiver operating characteristic curve (AUC-ROC).

Results: Xerophtalmia and xerostomia were presented in 185/205 (90.2\%) and $186 / 205(91.2 \%)$, respectively. According to ESSDAI, the majority of pSS patients $88 / 205(43 \%)$ had moderate disease activity. Seventy-eight per cent of pSS patients were anti-SSA antibody positive, 44\% anti-SSB/La antibody positive. Biopsy of LSG was positive in 140/172 (81.4\%) pSS patients. US abnormalities were established in $197(96 \%)$ pSS patients and in $16(18 \%)$ controls $(p<0.0001)$. Pathological sizes of salivary glands were more frequently in pSS patients than controls, $111(54.2 \%)$ vs. $3(3.4 \%)$ patients, respectively $(p<0.0001)$. The echogenicity of the salivary glands was pathological changes in $142(69.3 \%)$ pSS patients and in only $5(5.7 \%)$ control group $(\mathrm{p}<0.0001)$. The pathological glandular border was frequently in pSS patients than control group, $48(23.4 \%)$ vs. $2(2.3 \%), p<0.0001$. No differences were detected between the two groups of patients for enlarged intraglandular lymph nodes. Most of pSS patients had pathological inhomogeneity, 197/205 (96.1\%) vs. 16/85 (18.4\%) in control group $(p<0.0001)$. The median SGUS was significantly higher in pSS patients in comparison with control group [median (range) $4(0-6)$ vs. $0(0-2), p<0.0001]$. Forty-five percent of pSS patients had SGUS score 4 . The SGUS cut-off $\geq 2$ showed specificity of $89.5 \%$ and sensitivity $89.3 \%$. Diagnostic accuracy of the parenchymal inhomogeneity was very good (AUC-ROC 0.89 ), followed by the glandular echogenicity (AUC-ROC 0.81), the glandular size (AUC ROC 0.75), the posterior border (AUC ROC 0.60), and the presence of intraglandular lymph nodules (AUC ROC 0.49), respectively.

Conclusions: Our findings confirm that most of established pSS patients had pathological SGUS features. Among US parameters, parenchymal inhomogeneity was the most discriminant feature for diagnosis of SS. There is the growing evidence that ultrasound should be considered as the useful method for evaluation of salivary glands in pSS patients.

Disclosure of Interest: None declared

DOI: 10.1136/annrheumdis-2017-eular.5046

\section{THU0293 B-CELL RESPONSES TO TYPE I INTERFERON DEFINE DISEASE ACTIVITY IN SLE AND CAN BE MEASURED BY CELL SURFACE TETHERIN (CD317)}

Y.M. El-Sherbiny ${ }^{1,2}$, M.Y. Md Yusof ${ }^{1,2}$, A. Psarras ${ }^{2}$, E. Hensor ${ }^{1,2}$, R. Tooze ${ }^{3}$, G. Doody ${ }^{3}$, M. Wittmann ${ }^{1,2}$, P. Emery ${ }^{1,2}$, E.M. Vital ${ }^{1,2}{ }^{1}$ National Institute of Health Research Leeds Musculoskeletal Biomedical Research Unit, Leeds Teaching Hospitals NHS Trust; ${ }^{2}$ Leeds Institute of Rheumatic and Musculoskeletal Medicine; ${ }^{3}$ Leeds Institute of Cancer and Pathology, University of Leeds, Leeds, United Kingdom

Background: SLE is a Type I interferon (IFN-I) mediated disease with autoreactive $B$ cells. Whole blood interferon-stimulated gene (ISG) expression is used to measure IFN-I status but does not consistently correlate with clinical features and therapy response. ISG expression may be influenced by differing response in individual cell subsets as well as IFN-g. Tetherin is a cell surface protein encoded by the interferon-stimulated gene BST2.

Objectives: To evaluate tetherin expression as a novel cell-specific flow cytometric biomarker for IFN-I response.
Methods: In vitro, we tested response of expression of BST2 and 30 other ISGs to stimulation with IFN-a and IFN-g, as well as dose response of BST2 and tetherin protein in B cells. Sorted cells (Monocyte, T, NK, naïve and memory B, plasmablast) from 8 controls and 10 SLE patients were used to test variation in expression of 31 ISGs between cell subsets and whether tetherin measured by flow cytometry is a cell specific marker for IFN-I response. Samples from 156 SLE patients, 30 ACPA+ANA- RA (DAS28>3.2) patients and 22 healthy controls $(\mathrm{HC})$ were used in 3 clinical validation studies of tetherin vs. ISG score against diagnosis, disease activity (BILAG-2004) and plasmablast repopulation after rituximab. Results: Some ISGs' expression in B cells increased in response to both IFN-a and IFN-g. Others, which included BST2, were selective for IFN-a. An 18-gene ISG score derived from this latter group was calculated for comparison with tetherin.

Results from cell sorting showed that ISG Score was highest in monocytes; other subsets were $75-85 \%$ lower $(p<0.001)$. SLE-associated increase in expression (SLE:HC ratio) varied between 2.56 ( $\mathrm{T}$ cells) to 4.93 (plasmablasts).

Diagnosis: ISG score differentiated HC from SLE with ratio 3.58 (1.94 - 6.61) and large effect size 0.14 (partial eta squared). Using tetherin for cell specific IFN response revealed marked differences between subsets. Monocytes did not differentiate HC and SLE at all with ratio $1.19(0.87-1.61)$ and effect size 0.03 . Memory B cells had medium-large effect size of 0.11 with ratio $1.59(1.21-2.09)$. Comparing SLE and RA the largest effect size was for plasmablast Tetherin at plasmablasts at 0.23 , with ratio $2.20(1.66-2.93)$.

Disease activity: ISG score was associated with cutaneous disease activity (BILAG A/B) but not musculoskeletal (Fig 1). Monocyte tetherin was associated with musculoskeletal disease activity but not cutaneous. Memory B cell tetherin was associated with disease activity in both these organs. Memory B cell tetherin was increased with renal $(p=0.005)$ or haematological $(p=0.005)$ activity with no differences in ISG score for these domains ( $p=0.152, p=0.989$ respectively).

Plasmablast numbers after rituximab were associated with Memory B cell tetherin $(R=0.38, p=0.047)$ but not ISG score $(R=0.24, p=0.219)$.
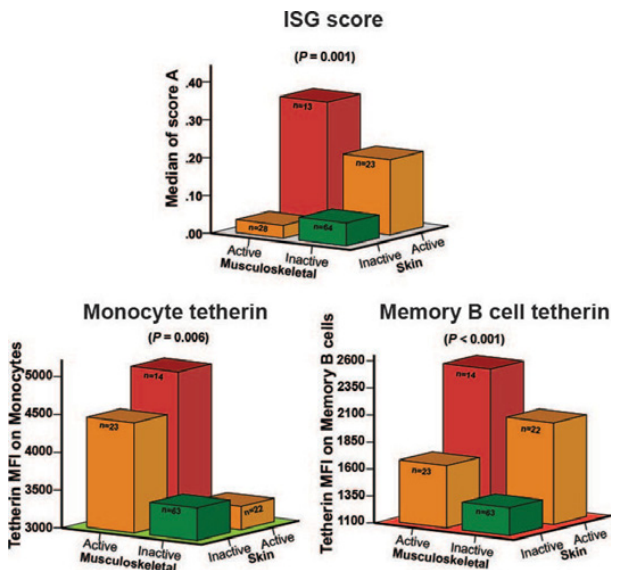

Conclusions: ISG expression in unsorted blood is influenced by IFN-g and cellular composition of the sample as well as IFN-I. Flow cytometric measurement of surface tetherin is a cell-specific assay for IFN-I that avoids these problems. Memory B cell tetherin was better associated with plasmablast numbers and clinical features of disease than monocyte tetherin or ISG score.

Disclosure of Interest: None declared

DOI: 10.1136/annrheumdis-2017-eular.6002

\section{THURSDAY, 15 JUNE 2017 Vasculitis}

\section{THU0294 DIFFERENTIAL PATTERNS OF ATROPHY IN HIPPOCAMPUS AND BRAINSTEM BETWEEN CHRONIC PROGRESSIVE NEURO-BEHCET'S DISEASE AND ALZHEIMER'S DISEASE}

H. Kikuchi ${ }^{1}$, K. Asako ${ }^{1}$, T. Yanagida ${ }^{1}$, H. Kono ${ }^{1}$, S. Hirohata ${ }^{2} .{ }^{1}$ Department of Internal Medicine, Teikyo University School of Medicine, Tokyo; ${ }^{2}$ Department of Rheumatology and Infectious Diseases, Kitasato University School of Medicine, Kanagawa, Japan

Background: Central nervous system involvement is one of the most serious complications in Behcet's disease (BD). This condition is referred to as neuroBehçet's disease (NB) and can be classified into acute type (ANB) and chronic progressive type (CPNB) based upon differences in the clinical course and responses to corticosteroid treatment. Cerebellar ataxia, such as gait disturbances and dysarthria, is one of the representative manifestations in CPNB. Accordingly, brainstem atrophy is frequently observed in CPNB, but not in ANB Notably, progressive neurobehavioral changes mimicking those in Alzheimer's disease (AD) are also frequently observed in CPNB, but these changes cannot be accounted for by brainstem atrophy. 